\title{
A Moving Mesh Numerical Method for Hyperbolic Conservation Laws*
}

\author{
By Bradley J. Lucier
}

\begin{abstract}
We show that the possibly discontinuous solution of a scalar conservation law in one space dimension may be approximated in $L^{1}(\mathbf{R})$ to within $O\left(N^{-2}\right)$ by a piecewise linear function with $O(N)$ nodes; the nodes are moved according to the method of characteristics. We also show that a previous method of Dafermos, which uses piecewise constant approximations, is accurate to $O\left(N^{-1}\right)$. These numerical methods for conservation laws are the first to have proven convergence rates of greater than $O\left(N^{-1 / 2}\right)$.
\end{abstract}

1. Introduction. It is well-known that the solution of the hyperbolic conservation law,

$$
\begin{gathered}
u_{t}+f(u)_{x}=0, \quad x \in \mathbf{R}, t>0, \\
u(x, 0)=u_{0}(x), \quad x \in \mathbf{R},
\end{gathered}
$$

may be calculated by the method of characteristics where the solution is smooth; elsewhere, shocks evolve according to the Rankine-Hugoniot and entropy conditions [16]. In this paper we transform the method of characteristics into a numerical algorithm by showing that if one carefully chooses $O(N)$ points to track by the method of characteristics, then for suitably smooth flux functions $f$ and piecewise smooth, but possibly discontinuous, initial data $u_{0}$, the error in the approximation is $O\left(N^{-2}\right)$ in $L^{1}(\mathbf{R})$. (For technical reasons our approximation may readily be calculated only for convex fluxes and certain problems with nonconvex fluxes.) Because our approximation is taken from the space of discontinuous piecewise linear functions, our method exhibits optimal-order accuracy $(1 / N$ may be considered a measure of the average mesh size). We also show that a previous method of Dafermos that uses a piecewise constant approximation has accuracy $O\left(N^{-1}\right)$, and hence is also optimal-order accurate. Although previous work has been done by others on formally second-order accurate methods that generate convergent approximations to solutions of $(\mathrm{C})$ for some fluxes $f$ (see [23], [8]), no other numerical method for scalar conservation laws has a proven convergence rate of more than $O\left(N^{-1 / 2}\right)$.

Received March 15, 1984; revised April 2, 1985.

1980 Mathematics Subject Classification. Primary 65M25, 35L65.

Key words and phrases. Conservation law, adaptive methods, method of characteristics.

*This material is based on work partially supported by the National Science Foundation under Grant No. DMS-8403219. 
There have been basically two approaches to designing adaptive methods for evolution equations, and specifically for conservation laws. (Hedstrom and Rodrigue [12] survey adaptive methods for evolution equations.) The first, which we will call the adaptive mesh approach, includes more meshpoints in the mesh wherever the approximate solution of (C) has large gradients; meshpoints are added and removed from the mesh when deemed necessary, but meshpoints are generally not moved from one timestep to the next, and the number of meshpoints may vary greatly over time. Oliger and his students [1], [2], [22], Lucier [18], and others (see [12]) have taken this approach. Osher and Sanders [24] have proved convergence for a method that uses this approach for conservation laws, but the rate of convergence of their method when used with any specific mesh selection algorithm is unknown.

The second, moving mesh, approach is to keep a more or less fixed number of meshpoints, and to move them according to a prescribed algorithm. Methods falling into this class include the moving finite-element methods of Miller [21] and Herbst et al. [13], which calculate the meshpoint positions as an integral part of the discrete weak formulation of the evolution equation; and the method introduced by Davis and Flaherty [6], who do a separate calculation at each time to determine a near-optimal grid on which to approximate the solution at that time. (A method may combine elements of each technique, of course.) One intriguing problem of justifying the moving-mesh approach is to show that even as shocks and regions of large gradients are forming in the solution, a predetermined number of meshpoints are sufficient to approximate the solution for all time. At least for convex fluxes, we show for our method that even though a smooth solution may develop shocks or discontinuous initial data should be smoothed into a continuous function with singularities, the meshpoints necessary for a certain level of approximation may be determined a priori.

Moving meshpoints along the characteristics is quite natural; see the references in [13] for previous methods using this idea. Although our method may be considered the next higher-order generalization of a method introduced by Dafermos [5], the direct motivation for our work was Miller's moving finite-element method and Dupont's analysis [7] of various moving-mesh algorithms for evolution equations that have smooth solutions. Dupont requires, typically, that the $L^{2}$-norms of the first derivatives of the solution of the evolution equation be finite, a condition that is not satisfied by the solutions of (C). Our method is also similar to a method of LeVeque [17] in which he approximates the solution of $(\mathrm{C})$ by studying the long time behavior of certain piecewise constant approximations to (C). (One can apply the techniques in this paper to obtain error estimates for certain variants of LeVeque's method; see [19].) We must remark that we deal only with the semidiscrete problem; although the system of differential equations that determine the evolution of the approximation solution may be solved in closed form, the implementation of the method is left for another report.

Harten and Hyman [9] introduced a constrained moving-mesh algorithm for systems of conservation laws; Sanders analyzed their method for the scalar equation [27]. By using an intelligent mesh positioning algorithm, Harten and Hyman achieved fairly spectacular computational results for various test problems in gas dynamics. (One difficulty with our method is that it is not obvious how to extend it 
to systems of equations.) Sanders' analysis proved convergence of such methods for scalar conservation laws when the underlying difference scheme is monotone, but he did not obtain a rate of convergence any better than that for fixed-mesh numerical schemes.

2. Approximation of Conservation Laws. Kružkov [14] provided existence and uniqueness results for certain classes of weak solutions of $(C)$ through the prescription of an entropy condition. The theory for solutions of $(\mathrm{C})$ used in this paper is expressed in the following theorem.

THEOREM 1 (KRUŽKov). If $f$ is locally Lipschitz continuous, then for any $u_{0} \in B V(\mathbf{R})$ (the class of function whose first derivatives are measures) and for any $T>0$ there is $a$ unique bounded $u \in B V(\mathbf{R} \times[0, T]) \cap C^{0}\left([0, T], L_{\mathrm{loc}}^{1}(\mathbf{R})\right)$ with $u(0)=u_{0}$ that satisfies the following entropy condition: for all $\phi \in C_{0}^{1}(\mathbf{R} \times \mathbf{R})$, with $\phi \geqslant 0$, for all $c \in \mathbf{R}$, and for all $t \in[0, T]$,

$$
\begin{aligned}
\iint_{\mathbf{R} \times[0, t]} & {\left[|u-c| \phi_{t^{\prime \prime}}+\operatorname{sgn}(u-c)(f(u)-f(c)) \phi_{x^{\prime \prime}}\right] d x^{\prime \prime} d t^{\prime \prime} } \\
& -\int_{\mathbf{R}}\left[\left|u\left(x^{\prime \prime}, t\right)-c\right| \phi\left(x^{\prime \prime}, t\right)-\left|u_{0}\left(x^{\prime \prime}\right)-c\right| \phi\left(x^{\prime \prime}, 0\right)\right] d x^{\prime \prime} \geqslant 0 .
\end{aligned}
$$

The analysis of monotone finite-difference schemes for scalar conversation laws is more or less complete (see [4], [10], [15], [20], [26]). The more successful results are based on Kuznetsov's general theory of approximation for solutions of (C) [15]. A formulation of Kuznetsov's theorem in one space dimension follows.

TheOREM 2 (KuzNetSov). Let $u$ be the entropy solution of $(\mathrm{C})$ with $u_{0} \in B V(\mathbf{R})$, and let $v: \mathbf{R}^{+} \rightarrow L_{\text {loc }}^{1}(\mathbf{R})$ have left and right limits for every $t$, and be right continuous. Pick a positive, symmetric function $\eta(\xi)$ with support in $[-1,1]$ and integral 1 , positive numbers $\varepsilon$ and $\varepsilon_{0}$, and let

$$
\omega(x, t)=\frac{1}{\varepsilon_{0}} \eta\left(\frac{t}{\varepsilon_{0}}\right) \frac{1}{\varepsilon} \eta\left(\frac{x}{\varepsilon}\right) .
$$

Define the "Kružkov form"

$$
\begin{aligned}
\Lambda_{t}^{\varepsilon_{0}, \varepsilon}= & \iint_{S \times S}\left|v\left(x^{\prime \prime}, t^{\prime \prime}\right)-u\left(x^{\prime}, t^{\prime}\right)\right| \frac{\partial}{\partial t^{\prime \prime}} \omega\left(x^{\prime \prime}-x^{\prime}, t^{\prime \prime}-t^{\prime}\right) \\
& +\operatorname{sgn}\left(v\left(x^{\prime \prime}, t^{\prime \prime}\right)-u\left(x^{\prime}, t^{\prime}\right)\right)\left(f\left(v\left(x^{\prime \prime}, t^{\prime \prime}\right)\right)-f\left(u\left(x^{\prime}, t^{\prime}\right)\right)\right) \\
\cdot \frac{\partial}{\partial x^{\prime \prime}} \omega\left(x^{\prime \prime}-x^{\prime}, t^{\prime \prime}-t^{\prime}\right) d x^{\prime \prime} d t^{\prime \prime} d x^{\prime} d t^{\prime} & \\
& +\int_{S \times \mathbf{R}}\left[\omega\left(x^{\prime \prime}-x^{\prime}, 0-t^{\prime}\right)\left|v_{0}\left(x^{\prime \prime}\right)-u\left(x^{\prime}, t^{\prime}\right)\right|\right. \\
& \left.-\omega\left(x^{\prime \prime}-x^{\prime}, t-t^{\prime}\right)\left|v\left(x^{\prime \prime}, t-0\right)-u\left(x^{\prime}, t^{\prime}\right)\right|\right] d x^{\prime \prime} d x^{\prime} d t^{\prime},
\end{aligned}
$$

where $S=\mathbf{R} \times(0, t)$. Then

$$
\begin{aligned}
\|u(t)-v(t)\|_{L^{1}(\mathbf{R})} \leqslant & \|u(0)-v(0)\|_{L^{1}(\mathbf{R})}+\left(2 \varepsilon+\|f\|_{L_{\text {ip }}} \varepsilon_{0}\right)\left|u_{0}\right|_{B V(\mathbf{R})} \\
& +\sup _{t^{\prime},|\tau|<\varepsilon_{0},-t^{\prime}<\tau<t-t^{\prime}}\left\|v\left(t^{\prime}+\tau\right)-v\left(t^{\prime}\right)\right\|_{L^{1}(\mathbf{R})}-\Lambda_{t}^{\varepsilon_{0},{ }^{*}},
\end{aligned}
$$

where $\|f\|_{\text {Lip }}=\sup _{x \neq y}|(f(x)-f(y)) /(x-y)|$. 
The following comparison theorem is the basis for our analysis.

THEOREM 3. If $f$ and $g$ are Lipschitz continuous functions, $u_{0}, v_{0} \in B V(\mathbf{R})$, and $u$ and $v$ are the solutions of

$$
\begin{gathered}
u_{t}+f(u)_{x}=0, \quad x \in \mathbf{R}, t>0, \\
u(x, 0)=u_{0}(x), \quad x \in \mathbf{R},
\end{gathered}
$$

and

$$
\begin{aligned}
v_{t}+g(v)_{x} & =0, \quad x \in \mathbf{R}, t>0, \\
v(x, 0) & =v_{0}(x), \quad x \in \mathbf{R},
\end{aligned}
$$

then for any $t>0$,

$$
\|u(t)-v(t)\|_{L^{1}(\mathbf{R})} \leqslant\left\|u_{0}-v_{0}\right\|_{L^{1}(\mathbf{R})}+t\|f-g\|_{\text {Lip }} \min \left(\left|u_{0}\right|_{B V(\mathbf{R})},\left|v_{0}\right|_{B V(\mathbf{R})}\right) .
$$

Proof. We use Kuznetsov's theorem to compare $v$ to $u$. Kružkov [14] shows that

$$
\sup _{t^{\prime},|\tau|<\varepsilon_{0},-t^{\prime}<\tau<t-t^{\prime}}\left\|v\left(t^{\prime}+\tau\right)-v\left(t^{\prime}\right)\right\|_{L^{1}(\mathbf{R})} \leqslant \varepsilon_{0}\|g\|_{\text {Lip }}\left|v_{0}\right|_{B V(\mathbf{R})} .
$$

To bound $-\Lambda_{t}^{\varepsilon_{0}, \varepsilon}$, one may use the fact that $v$ satisfies the inequality of Theorem 1 with the flux $g$, so that after setting $c=u\left(x^{\prime}, t^{\prime}\right), \phi\left(x^{\prime \prime}, t^{\prime \prime}\right)=\omega\left(x^{\prime \prime}-x^{\prime}, t^{\prime \prime}-t^{\prime}\right)$, and integrating (1) over $x^{\prime}, t^{\prime}$, one only has to bound (after an integration by parts)

$$
\begin{aligned}
\iint_{S \times S} \frac{\partial}{\partial x^{\prime \prime}}\left[\operatorname{sgn}\left(v\left(x^{\prime \prime}, t^{\prime \prime}\right)-u\left(x^{\prime}, t^{\prime}\right)\right)((f-\right. & g)\left(v\left(x^{\prime \prime}, t^{\prime \prime}\right)\right) \\
& \left.\left.-(f-g)\left(u\left(x^{\prime}, t^{\prime}\right)\right)\right)\right] \\
& \cdot \omega\left(x^{\prime \prime}-x^{\prime}, t^{\prime \prime}-t^{\prime}\right) d x^{\prime \prime} d t^{\prime \prime} d x^{\prime} d t^{\prime} .
\end{aligned}
$$

Because $v(t) \in B V(\mathbf{R})$ for each $t$, we can find an increasing function $v(\cdot, t)^{+}$and a decreasing function $v(\cdot, t)^{-}$so that $v(\cdot, t)=v(\cdot, t)^{+}+v(\cdot, t)^{-}$. Define $v(\cdot, t)^{t}=$ $v(\cdot, t)^{+}-v(\cdot, t)^{-}$, and let $h=f-g$. Now, because $\operatorname{sgn}(a-b)(h(a)-h(b))=$ $h(a \vee b)-h(a \wedge b)$, where $a \vee b=\max (a, b)$ and $a \wedge b=\min (a, b)$, a simple case analysis shows that

$$
\begin{aligned}
& \mid \operatorname{sgn}(\left.\left(x_{1}^{\prime \prime}, t^{\prime \prime}\right)-u\left(x^{\prime}, t^{\prime}\right)\right)\left(h\left(v\left(x_{1}^{\prime \prime}, t^{\prime \prime}\right)-h\left(u\left(x^{\prime}, t^{\prime}\right)\right)\right) \mid\right. \\
&-\operatorname{sgn}\left(v\left(x_{2}^{\prime \prime}, t^{\prime \prime}\right)-u\left(x^{\prime}, t^{\prime}\right)\right)\left(h\left(v\left(x_{2}^{\prime \prime}, t^{\prime \prime}\right)\right)-h\left(u\left(x^{\prime}, t^{\prime}\right)\right)\right) \mid \\
& \leqslant\left|h\left(v\left(x_{1}^{\prime \prime}, t^{\prime \prime}\right) \vee u\left(x^{\prime}, t^{\prime}\right)\right)-h\left(v\left(x_{2}^{\prime \prime}, t^{\prime \prime}\right) \vee u\left(x^{\prime}, t^{\prime}\right)\right)\right| \\
& \quad+\left|h\left(v\left(x_{1}^{\prime \prime}, t^{\prime \prime}\right) \wedge u\left(x^{\prime}, t^{\prime}\right)\right)-h\left(v\left(x_{2}^{\prime \prime}, t^{\prime \prime}\right) \wedge u\left(x^{\prime}, t^{\prime}\right)\right)\right| \\
& \leqslant\|h\|_{\text {Lip }}\left|v^{t}\left(x_{1}^{\prime \prime}, t^{\prime \prime}\right)-v^{t}\left(x_{2}^{\prime \prime}, t^{\prime \prime}\right)\right| .
\end{aligned}
$$

Therefore,

$$
\left|\frac{\partial}{\partial x^{\prime \prime}}\left[\operatorname{sgn}\left(v\left(x^{\prime \prime}, t^{\prime \prime}\right)-u\left(x^{\prime}, t^{\prime}\right)\right)(f-g)\left(v\left(x^{\prime \prime}, t^{\prime \prime}\right)-u\left(x^{\prime}, t^{\prime}\right)\right)\right]\right|
$$

is bounded, as a measure, by $\|f-g\|_{\text {Lip }}\left|\left(\partial v / \partial x^{\prime \prime}\right)\left(x^{\prime \prime}, t^{\prime \prime}\right)\right|$. Substituting this expression in (3), we may bound $-\Lambda_{t}^{\varepsilon_{0}, \varepsilon}$ by the integral of the convolution of the measures $\|f-g\|_{\text {Lip }}\left|v_{x}\right|$ and $\omega$. Because $|v(t)|_{B V(\mathbf{R})} \leqslant\left|v_{0}\right|_{B V(\mathbf{R})}$ for all $t$, and $\omega$ has integral one, the integral (3) is bounded by $t\|f-g\|_{\text {Lip }}\left|v_{0}\right|_{B V(\mathbf{R})}$. Let $\varepsilon$ and $\varepsilon_{0}$ tend to zero. The final result follows because of symmetry. 
3. Application to Dafermos's Method. Dafermos's method [5], which was tested on both scalar equations and systems by Hedstrom [11], is described below. Let $N$ be a positive integer. If the flux $f$ is $C^{2}$ (a modified argument shows that $f$ need only be Lipschitz continuous and piecewise $C^{2}$ ), then define the continuous, piecewise linear (with breakpoints at $j / N$ for all $j$ ) function $f^{\varepsilon}$ by $f^{\varepsilon}(j / N)=f(j / N)$ for every integer $j$. Then $\left\|f^{\varepsilon}-f\right\|_{\text {Lip }} \leqslant(2 N)^{-1}\left\|f^{\prime \prime}\right\|_{L^{\infty}(\mathbf{R})}$. If $u_{0}$ consists of a finite number of constant states, then Dafermos gives the entropy solution of

$$
\begin{aligned}
u_{t}^{\varepsilon}+f^{\varepsilon}\left(u^{\varepsilon}\right)_{x} & =0, \quad x \in \mathbf{R}, t>0, \\
u^{\varepsilon}(x, 0) & =u_{0}(x), \quad x \in \mathbf{R},
\end{aligned}
$$

in the following way. Dafermos first reduces the problem to a Riemann problem, because the initial datum has finitely many jumps. If $u_{0}$ is specified as

$$
u_{0}(x)= \begin{cases}u_{l}, & x \leqslant 0, \\ u_{r}, & x>0,\end{cases}
$$

with $u_{l}<u_{r}$, then the vertices of the boundary of the convex hull of $\left\{(u, v) \mid u_{l} \leqslant\right.$ $\left.u \leqslant u_{r}, \quad v \geqslant f^{\varepsilon}(u)\right\}$ will consist of a set of points $\left(u_{l}, f^{\varepsilon}\left(u_{l}\right)\right),\left(u^{1}, f^{\varepsilon}\left(u^{1}\right)\right), \ldots$, $\left(u^{k}, f^{\varepsilon}\left(u^{k}\right)\right),\left(u_{r}, f^{\varepsilon}\left(u_{r}\right)\right)$, where $\left\{u^{i}\right\}$ is a linearly ordered subset of $\{j / N\}$. The solution will then be given by the following set of constant states:

$$
\begin{aligned}
u^{\varepsilon}(x, t) \equiv & u_{l} \quad \text { for }-\infty<\frac{x}{t} \leqslant \frac{f^{\varepsilon}\left(u^{1}\right)-f^{\varepsilon}\left(u_{l}\right)}{u^{1}-u_{l}}, \\
& u^{1} \quad \text { for } \frac{f^{\varepsilon}\left(u^{1}\right)-f^{\varepsilon}\left(u_{l}\right)}{u^{1}-u_{l}}<\frac{x}{t} \leqslant \frac{f^{\varepsilon}\left(u^{2}\right)-f^{\varepsilon}\left(u^{1}\right)}{u^{2}-u^{1}}, \\
& \vdots \\
& u^{k} \quad \text { for } \frac{f^{\varepsilon}\left(u^{k}\right)-f^{\varepsilon}\left(u^{k-1}\right)}{u^{k}-u^{k-1}}<\frac{x}{t} \leqslant \frac{f^{\varepsilon}\left(u_{r}\right)-f^{\varepsilon}\left(u^{k}\right)}{u_{r}-u^{k}}, \\
& u_{r} \text { for } \frac{f^{\varepsilon}\left(u_{r}\right)-f^{\varepsilon}\left(u^{k}\right)}{u_{r}-u^{k}}<\frac{x}{t}<\infty .
\end{aligned}
$$

A similar result may be inferred if $u_{l}>u_{r}$ by considering the convex hull of the set $\left\{(u, v) \mid u_{r} \leqslant u \leqslant u_{l}, v \leqslant f^{\varepsilon}(u)\right\}$. Thus, for all time, the approximate solution $u^{\varepsilon}$ will take on only finitely many states (no more than $N\left\|u_{0}\right\|_{B V(\mathbf{R})}$ plus twice the initial number of states). Theorem 3 implies that

$$
\left\|u-u^{\varepsilon}\right\|_{L^{1}(\mathbf{R})} \leqslant \frac{t}{2 N}\left\|f^{\prime \prime}\right\|_{L^{\infty}(\mathbf{R})}\left|u_{0}\right|_{B V(\mathbf{R})} .
$$

Thus, Dafermos's method is first-order accurate. The error's linear growth in time is consistent with Hedstrom's numerical results.

Dafermos mentions that there is no known proof that only a finite number of wave interactions occur in any finite time interval if $f^{\varepsilon}$ is not convex; however, I believe that this is the case for the special form of the flux $f^{\varepsilon}$ and solution $u^{\varepsilon}$.

4. A Method Based on Piecewise Linear Elements. We now assume that the flux $f$ has three bounded derivatives. The initial data $u_{0}$ will be restricted to the class of functions in $B V(\mathbf{R})$ whose first derivatives are also in $B V(\mathbf{R})$ outside a finite set of 
points $\left\{s_{i}\right\}$. We assume that the support of $u_{0}$ is contained in $[-1,1]$ and that the range of $u_{0}$ is contained in $[0,1]$. (Generalizations to initial data that is constant outside an interval will be obvious.) Throughout the following, $\varepsilon$ will denote $1 / N, N$ being an integer greater than one. Let $\delta=\min _{i, j}\left(\varepsilon^{2},\left|s_{i}-s_{j}\right| / 2\right)$.

The function $f$ is approximated by a parabolic spline approximation $f^{\varepsilon}$, a continuously differentiable, piecewise quadratic function whose second derivative is discontinuous at the breakpoints $\eta_{k}=k / N$ for $k=0,1, \ldots, N$. The function $f^{\varepsilon}$ is the unique parabolic spline that has $\left(d f^{\varepsilon} / d x\right)\left(\eta_{k}\right)=f^{\prime}\left(\eta_{k}\right)$, with $d f^{\varepsilon} / d x$ a linear function between $\eta_{k}$ and $\eta_{k+1}$, and $f^{\varepsilon}(0)=0$. Then $\left\|f^{\varepsilon}-f\right\|_{\text {Lip }} \leqslant \frac{1}{4}\left\|f^{\prime \prime \prime}\right\|_{L^{\infty}(\mathbf{R})} \varepsilon^{2}$ (see [3]).

The function $u_{0}^{\varepsilon}$ and the initial meshpoints $\xi_{i}$ are to be chosen as follows.

(a) Modify $u_{0}$ near each discontinuity $s_{i}$ by redefining it as a continuous linear function between $s_{i}-\delta$ and $s_{i}+\delta$, which we will take as our first meshpoints.

(b) In the intervals where $u_{0}$ is smooth, insert a minimal number of meshpoints so that

$$
\left|\xi_{i+1}-\xi_{i}\right| \int_{\left(\xi_{i}, \xi_{i+1}\right)}\left|u_{0}^{\prime \prime}\right| d x \leqslant \varepsilon^{2}\left|\frac{d u_{0}}{d x}\right|_{B V\left(\mathbf{R} \backslash\left\{s_{i}\right\}\right)} .
$$

(c) Set $u_{0}^{\varepsilon}\left(\xi_{i}\right)=u_{0}\left(\xi_{i}\right)$ for all $i$, and let $u_{0}^{\varepsilon}$ be a linear function between the meshpoints $\xi_{i}$.

(d) Insert new meshpoints $\xi_{i}$ at the points where $u_{0}^{\varepsilon}\left(\xi_{i}\right)=\eta_{k}$, for all $k$.

This approximation ensures that

$$
\left\|u_{0}^{\varepsilon}-u_{0}\right\|_{L^{1}(\mathbf{R})} \leqslant\left(\left|u_{0}\right|_{B V(\mathbf{R})}+\left|\frac{d u_{0}}{d x}\right|_{B V\left(\mathbf{R} \backslash\left\{s_{1}\right\}\right)}\right) \varepsilon^{2}
$$

(see [3]).

THEOREM 4. There are fewer than $4 k+\left(\left|u_{0}\right|_{B V(\mathbf{R})}+2\right) N$ meshpoints $\xi_{i}$, where $u_{0}$ has $k$ discontinuities.

Proof. Step (a) starts the mesh with $2 k$ points. It is known [3] that step (b) introduces no more than $N$ meshpoints. Consider now only the meshpoints $\xi_{i}$ added by step (d), order them separately, and call them $\nu_{i}$. At most $2 k+N$ intervals $\left[\nu_{i}, \nu_{i+1}\right]$ may contain previously inserted meshpoints, and if $\left[\nu_{i}, \nu_{i+1}\right]$ contains no old meshpoints, then $\left|u_{0}^{\varepsilon}\left(\nu_{i}\right)-u_{0}^{\varepsilon}\left(\nu_{i+1}\right)\right|=1 / N$, because $u_{0}^{\varepsilon}$ is linear between the original meshpoints. Thus, step (d) adds at most $\left(\left|u_{0}\right|_{B V(\mathbf{R})}+1\right) N+2 k$ meshpoints $\nu_{i}$.

The solution of the approximate problem,

$$
\begin{gathered}
u_{t}^{\varepsilon}+f^{\varepsilon}\left(u^{\varepsilon}\right)_{x}=0, \quad x \in \mathbf{R}, t>0, \\
u^{\varepsilon}(x, 0)=u_{0}^{\varepsilon}(x), \quad x \in \mathbf{R},
\end{gathered}
$$

may now be calculated, for example, by the method of characteristics. Because $f^{\varepsilon}$ is quadratic on the range of $u^{\varepsilon}$ in each interval $\left(\xi_{i}(t), \xi_{i+1}(t)\right)$ where $u^{\varepsilon}$ is continuous, $u^{\varepsilon}$ is linear on that interval, and only the nodal values need be determined. The evolution of shocks may again be determined by the Rankine-Hugoniot condition, as long as the entropy condition is verified. In particular, $u^{\varepsilon}(x, t)$ may be found as follows. Let $u^{\varepsilon}(x, t)$ have left and right limits of $l_{i}(t)$ and $r_{i}(t)$ at the meshpoint $\xi_{i}(t)$. Then $l_{i}, r_{i}$, and $\xi_{i}$ satisfy the following differential equations (for clarity, we 
write $f$ in place of $f^{\varepsilon}$ ):

$$
\begin{gathered}
\frac{d \xi_{i}}{d t}=\left\{\begin{array}{l}
f^{\prime}\left(l_{i}(t)\right) \quad \text { if } l_{i}(t)=r_{i}(t), \\
\frac{f\left(l_{i}(t)\right)-f\left(r_{i}(t)\right)}{l_{i}(t)-r_{i}(t)} \text { if } l_{i}(t) \neq r_{i}(t),
\end{array}\right. \\
\frac{d l_{i}}{d t}= \begin{cases}0 \quad \text { if } l_{i}(t)=r_{i}(t), \\
\frac{l_{i}(t)-r_{i-1}(t)}{\xi_{i}(t)-\xi_{i-1}(t)}\left(\frac{f\left(l_{i}(t)\right)-f\left(r_{i}(t)\right)}{l_{i}(t)-r_{i}(t)}-f^{\prime}\left(l_{i}(t)\right)\right)\end{cases} \\
\frac{d r_{i}}{d t}=\left\{\begin{array}{ll}
0 \quad \text { if } l_{i}(t)=r_{i}(t), & \text { if } l_{i}(t) \neq r_{i}(t), \\
-\frac{l_{i+1}(t)-r_{i}(t)}{\xi_{i+1}(t)-\xi_{i}(t)}\left(\frac{f\left(l_{i}(t)\right)-f\left(r_{i}(t)\right)}{l_{i}(t)-r_{i}(t)}-f^{\prime}\left(r_{i}(t)\right)\right.
\end{array}\right) \\
\end{gathered}
$$

Note that most of the equations are trivial; whenever $u^{\varepsilon}$ is continuous at $\xi_{i}(t)$, $d \xi_{i} / d t$ is constant, while $d r_{i} / d t$ and $d l_{i} / d t$ are 0 . That is, the meshpoint moves along the characteristic of the perturbed equation, and the approximate solution is constant along the characteristic. Even where there is a shock in the approximate solution, the system of equations may be solved in closed form between shock interaction times. The behavior of $u^{\varepsilon}$ on either side of a shock is easy to determine, and one only needs to find the trajectory of the meshpoint $\xi_{i}$. With the help of the symbolic manipulation program Macsyma, I used the fact that mass is conserved near the discontinuity to find that the shock trajectory is given as part of the zero set of a polynomial $P(x, t)=0$ of total degree three. The coefficients of the polynomial are given in Table 1. To the left of the shock, the approximate flux has the value $f^{\varepsilon}(u)=a_{l} u^{2}+2 b_{l} u+c_{l}$, and $u^{\varepsilon}$ has initial slope $s_{l}$; similarly to the right of the shock. The initial shock position and the left and right limits of $u^{\varepsilon}(x, 0)$ at the shock are given by $\xi_{i}, l_{i}$, and $r_{i}$.

The entropy condition must be checked for the solution $u^{\varepsilon}$, however. That is, if $u^{\varepsilon}$ is discontinuous at $\xi_{i}(t)$, then

$$
\frac{f^{\varepsilon}\left(l_{i}(t)\right)-f^{\varepsilon}\left(r_{i}(t)\right)}{l_{i}(t)-r_{i}(t)} \geqslant \frac{f^{\varepsilon}(\zeta)-f^{\varepsilon}\left(r_{i}(t)\right)}{\zeta-r_{i}(t)} \text { for } \zeta \in\left[r_{i}(t), l_{i}(t)\right]
$$

if $l_{i}(t)>r_{i}(t)$, or

$$
\frac{f^{\varepsilon}\left(r_{i}(t)\right)-f^{\varepsilon}\left(l_{i}(t)\right)}{r_{i}(t)-l_{i}(t)} \leqslant \frac{f^{\varepsilon}(\zeta)-f^{\varepsilon}\left(l_{i}(t)\right)}{\zeta-l_{i}(t)} \text { for } \zeta \in\left[l_{i}(t), r_{i}(t)\right]
$$

if $r_{i}(t)>l_{i}(t)$. It is not difficult to show that if $f$ is convex, then $f^{\varepsilon}$ is convex, and, because $u_{0}^{\varepsilon}$ is continuous, the entropy condition will be trivially satisfied for any solution of the above system of differential equations, and no new meshpoints are introduced during the evolution of $u^{\varepsilon}$. There are cases when $f$ is not convex where the entropy condition is trivial to verify for certain data; see the second example below. We are led to the following theorem. 
TABLE 1

Coefficients for discontinuity curve $P(x, t)=0$

\begin{tabular}{|c|c|}
\hline$x^{2} t$ & $2\left(a_{r}-a_{l}\right) s_{l} s_{r}$ \\
\hline$x t^{2}$ & $8\left(a_{l} b_{r}-a_{r} b_{l}\right) s_{l} s_{r}$ \\
\hline$t^{3}$ & $8\left(a_{l} a_{r}\left(c_{r}-c_{l}\right)+a_{r} b_{l}^{2}-a_{l} b_{r}^{2}\right) s_{l} s_{r}$ \\
\hline$x^{2}$ & $s_{l}-s_{r}$ \\
\hline$x t$ & $\begin{array}{l}4\left[\left(a_{l}-a_{r}\right) s_{l} s_{r} \xi_{i}\right. \\
\left.\quad+\left(a_{r} l_{i} s_{r}-a_{l} r_{i} s_{l}\right)+\left(b_{r} s_{r}-b_{l} s_{l}\right)\right]\end{array}$ \\
\hline$t^{2}$ & $\begin{array}{l}8\left(a_{r} b_{l}-a_{l} b_{r}\right) s_{l} s_{r} \xi_{i}+4\left(b_{l}^{2} s_{l}-b_{r}^{2} s_{r}\right) \\
\quad+4 a_{l} a_{r}\left(r_{i}^{2} s_{l}-l_{i}^{2} s_{r}\right)+8\left(a_{l} b_{r} r_{i} s_{l}-a_{r} b_{l} l_{i} s_{r}\right) \\
\quad+4\left(a_{r} c_{r} s_{r}-a_{r} c_{l} s_{r}+a_{l} c_{r} s_{l}-a_{l} c_{l} s_{l}\right)\end{array}$ \\
\hline$x$ & $2\left(s_{r}-s_{l}\right) \xi_{i}+2\left(l_{i}-r_{i}\right)$ \\
\hline$t$ & $\begin{array}{l}2\left(a_{r}-a_{l}\right) s_{l} s_{r} \xi_{i}^{2} \\
\quad+4\left(a_{l} r_{i} s_{l}-a_{r} l_{i} s_{r}+b_{l} s_{l}-b_{r} s_{r}\right) \xi_{i} \\
\quad+2\left(f^{\varepsilon}\left(r_{i}\right)-f^{\varepsilon}\left(l_{i}\right)\right)\end{array}$ \\
\hline 1 & $\left(s_{l}-s_{r}\right) \xi_{i}^{2}+2\left(r_{i}-l_{i}\right) \xi_{i}$ \\
\hline
\end{tabular}

THEOREM 5. Let $f \in W_{\infty}^{3}(\mathbf{R})$ be convex, and let $u_{0} \in B V(\mathbf{R})$, with $u_{0}^{\prime}$ in $B V(\mathbf{R})$ outside $k$ points $\left\{s_{i}\right\}$. If $u(x, t)$ is the entropy solution of $(\mathrm{C})$, then by choosing $4 k+\left(\left|u_{0}\right|_{B V(\mathbf{R})}+2\right) N$ meshpoints $\xi_{i}$ and solving the algebraic-differential system $(5 \mathrm{a}-\mathrm{e})$ with initial data $\xi_{i}(0), r_{i}(0)$, and $l_{i}(0)$ derived from $u_{0}^{\varepsilon}$, we ensure that

$$
\begin{aligned}
& \left\|u^{\varepsilon}(\cdot, t)-u(\cdot, t)\right\|_{L^{1}(\mathbf{R})} \\
& \quad \leqslant\left[\left|u_{0}\right|_{B V(\mathbf{R})}+\left|\frac{d u_{0}}{d x}\right|_{B V\left(\mathbf{R} \backslash\left(s_{i}\right\}\right)}+\frac{1}{4}\left|u_{0}\right|_{B V(\mathbf{R})}\left\|f^{\prime \prime \prime}\right\|_{L^{\infty}(\mathbf{R})} t\right] / N^{2} .
\end{aligned}
$$

Because $u^{\varepsilon}$ is specified in terms of $O(N)$ parameters, this algorithm generates a second-order approximation to $u$.

Note that the error estimate applies for nonconvex $f$ as well; the only questions that arise are whether more meshpoints are generated during the evolution of $u^{\varepsilon}$, and whether infinitely many wave interactions may occur in a finite time interval. We believe that both statements are false for the special form of $f^{\varepsilon}$ given above.

It is interesting to note that the above node placement strategy is not optimal for higher-order approximations. Specifically, consider the problem,

$$
u_{t}+\left(u^{3}\right)_{x}=0, \quad x \in \mathbf{R}, t>0,
$$

with initial data that is 1 for $x \leqslant 0,1-x$ for $0 \leqslant x \leqslant 1$, and 0 for $x \geqslant 1$. Then,

$$
u(x, 1 / 6)= \begin{cases}1, & x \leqslant 1 / 2, \\ 1-\sqrt{2 x-1}, & 1 / 2 \leqslant x \leqslant 1, \\ 0, & 1 \leqslant x\end{cases}
$$


If the nodes are initially placed at the points $j / N$, for $j$ between 0 and $N$, then the nodes at time $1 / 6$ are placed at $\left(1+j^{2} / N^{2}\right) / 2$ for $j$ between 0 and $N$. One may then calculate $\bar{u}$, the best $L^{1}(\mathbf{R})$ approximation to $u(\cdot, 1 / 6)$, using continuous piecewise quadratic elements with the additional requirement that $\bar{u}(x)=u(x)$ whenever $x$ is a node (see Rice [25, p. 102]). The first term in the asymptotic series in $N$ of $\|u(\cdot, 1 / 6)-\bar{u}\|_{L^{1}(\mathbf{R})}$ is

$$
\frac{1}{16 N^{3}} \sum_{j=0}^{N-1} \frac{1}{2 j+1}
$$

This calculation shows that the best $L^{1}(\mathbf{R})$ approximation with the given nodes has error $O\left(N^{-3} \log N\right)$, and not $O\left(N^{-3}\right)$, which is possible with a different node placement (see [3]). Thus, the node placement algorithm is not adequate to approximate the given singularity within the class of piecewise quadratic functions.

5. Computational Results. Two examples illustrate the placement of the meshpoints by our algorithm. The first example, illustrated in Figure 1, is the solution of

$$
\begin{gathered}
u_{t}+\left(u^{3}\right)_{x}=0, \quad x \in \mathbf{R}, t>0, \\
u(x, 0)=\chi_{[0,1]}(x), \quad x \in \mathbf{R},
\end{gathered}
$$

up to time $1 / 4$ with $N=20$. For this problem, we have

$$
\left|u_{0}\right|_{B V(\mathbf{R})}=2, \quad\left|d u_{0} / d x\right|_{B V\left(\mathbf{R} \backslash\left\{s_{i}\right\}\right)}=0, \quad \text { and }\left\|f^{\prime \prime \prime}\right\|_{L^{\infty}(\mathbf{R})}=6 .
$$

We can conclude that $\left\|u(t)-u^{\varepsilon}(t)\right\|_{L^{1}(\mathbf{R})}=(2+3 t) \varepsilon^{2}$; the error is less than 0.007 .

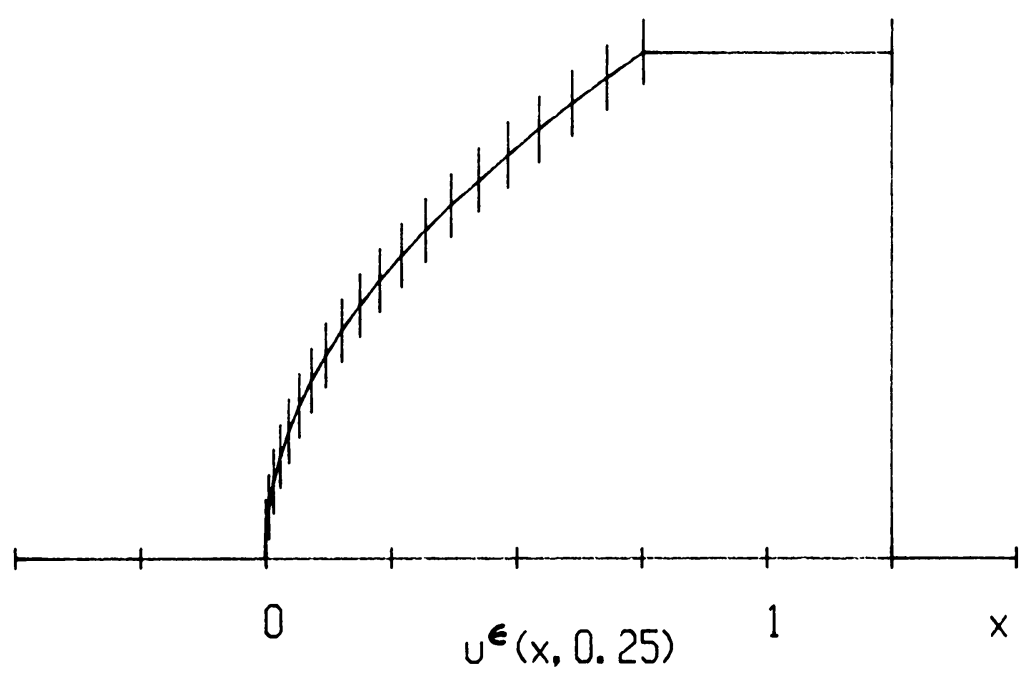

FIGURE 1

The graph of $u^{\varepsilon}(x, 1 / 4)$ for the first example (see text). The tick marks indicate the position of the nodes, between which the solution is linear. 


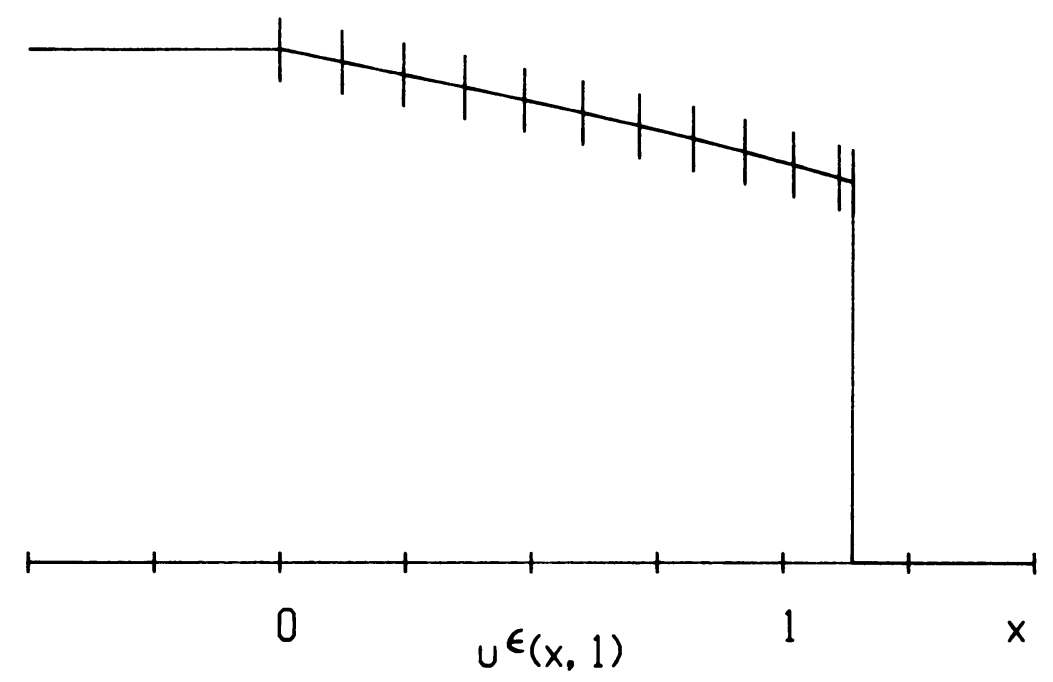

FIGURE 2

The graph of $u^{\varepsilon}(x, 1)$ for the second example (see text). The tick marks indicate the position of the nodes, between which the solution is linear.

The second example (Figure 2) has $f(u)=\frac{1}{2}(1-\cos (\pi u))$ and $u_{0}=\chi_{(-\infty, 0]}$. This flux is not convex, but the only difficulty in determining the numerical solution is in calculating the location of the shock. By conservation of mass, one places the shock so that the integral of $u^{\varepsilon}$ between $-\varepsilon^{2}$ and the position of the shock, in which region $u^{\varepsilon}$ may be calculated from (5), is equal to $\varepsilon^{2}+\left(f^{\varepsilon}(1)-f^{\varepsilon}(0)\right) t$. In this example, $N$ is 40 and $t$ is 1 . We have incurred a mass error equal to $\left(f(1)-f^{\varepsilon}(1)\right) t$, which is of the order of $\varepsilon^{2} t$. The same level of approximation may be achieved without a mass error by using the modified approximate flux $\overline{f^{\epsilon}}(u)=$ $f^{\varepsilon}(u) f(1) / f^{\varepsilon}(1)$.

Acknowledgments. Jerry Bona and Todd Dupont offered several suggestions that considerably improved this paper. The equation of the discontinuity curve was discovered during work with Fabio Milner on implementing the numerical scheme.

Department of Mathematics

Purdue University

West Lafayette, Indiana 47907

1. M. Berger, Adaptive Mesh Refinement for Hyperbolic Partial Differential Equations, Stanford Computer Science Report STAN-CS-82-924 (dissertation).

2. J. H. Bolstad, An Adaptive Finite Difference Method for Hyperbolic Systems in One Space Dimension, Lawrence Berkeley Lab. LBL-13287 (STAN-CS-82-899) (dissertation).

3. C. DE BOoR, "Good approximation by splines with variable knots," in Spline Functions and Approximation Theory (A. Meir and A. Sharma, eds.), ISNM, v. 21, Birkhäuser-Verlag, Basel, 1973, pp. 57-72.

4. M. G. Crandall \& A. Majda, "Monotone difference approximations for scalar conservation laws," Math. Comp., v. 34, 1980, pp. 1-21.

5. C. M. Dafermos, "Polygonal approximations of solutions of the initial value problem for a conservation law,” J. Math. Anal. Appl., v. 38, 1972, pp. 33-41. 
6. S. F. Davis \& J. E. Flaherty, "An adaptive finite element method for initial-value problems for partial differential equations,” SIAM J. Sci. Statist. Comput., v. 3, 1982, pp. 6-28.

7. T. DuPOnt, "Mesh modification for evolution equations," Math. Comp., v. 39, 1982, pp. 85-107.

8. A. Harten, “High resolution schemes for hyperbolic conservation laws," J. Comput. Phys., v. 49. 1983, pp. $357-393$.

9. A. Harten \& J. M. Hyman, "Self-adjusting grid methods for one-dimensional hyperbolic conservation laws," J. Comput. Phys., v. 50, 1983, pp. 235-269.

10. A. Harten, J. M. Hyman \& P. D. LaX, "On finite difference approximations and entropy conditions for shocks," Comm. Pure Appl. Math., v. 29, 1976, pp. 297-322.

11. G. W. Hedstrom, "Some numerical experiments with Dafermos's method for nonlinear hyperbolic equations," Lecture Notes in Math., vol. 267, Springer-Verlag, Berlin and New York, 1972, pp. 117-138.

12. G. W. Hedstrom \& G. H. Rodrigue, "Adaptive-grid methods for time-dependent partial differential equations," in Multigrid Methods (W. Hackbusch and U. Trottenberg, eds.), Springer-Verlag, Berlin and New York, 1982, pp. 474-484.

13. B. M. Herbst, S. W. Schoombie \& A. R. Mitchell, "Equidistributing principles in moving finite element methods," J. Comput. Appl. Math., v. 9, 1983, pp. 377-489.

14. S. N. KRUŽKov, "First order quasilinear equations with several space variables," Math. USSR Sb., v. 10,1970 , pp. $217-243$.

15. N. N. KuZnetsov, "Accuracy of some approximate methods for computing the weak solutions of a first-order quasi-linear equation," USSR Comput. Math. and Math. Phys., v. 16, no. 6, 1976, pp. 105-119.

16. P. D. Lax, Hyperbolic Systems of Conservation Laws and the Mathematical Theory of Shock Waves, SIAM Regional Conference Lectures in Applied Mathematics, no. 11, 1972.

17. R. LEVEQUE, "Large time step shock-capturing techniques for scalar conservation laws," SIAM J. Numer. Anal., v. 19, 1982, pp. 1091-1109.

18. B. J. LUCIER, "A stable adaptive numerical scheme for hyperbolic conservation laws," SIAM J. Numer. Anal., v. 22, 1985, pp. 180-203.

19. B. J. LuCIER, "Error bounds for the methods of Glimm, Godunov, and LeVeque," SIAM J. Numer. Anal., Dec. 1985.

20. B. J. LUCIER, “On nonlocal monotone difference methods for scalar conservation laws," Math. Comp. (To appear.)

21. K. Miller, “Moving finite elements, part II,” SIAM J. Numer. Anal., v. 18, 1981, pp. 1019-1057.

22. J. Oliger, "Approximate methods for atmospheric and oceanographic circulation problems," Lecture Notes in Physics, vol. 91 (R. Glowinski and J. Lions, eds.), Springer-Verlag, Berlin and New York, 1979, pp. 171-184.

23. S. Osher \& S. Chakravarthy, High Resolution Schemes and the Entropy Condition, ICASE Report 172218.

24. S. OShER \& R. SANDERS, "Numerical approximations to nonlinear conservation laws with locally varying time and space grids," Math. Comp., v. 41, 1983, pp. 321-336.

25. J. R. Rice, The Approximation of Functions, Vol. 1, Addison-Wesley, Reading, Mass., 1964.

26. R. SANDERS, "On convergence of monotone finite difference schemes with variable spatial differencing," Math. Comp., v. 40, 1983, pp. 91-106.

27. R. SANDERS, "The moving grid method for nonlinear hyperbolic conservation laws," SIAM J. Numer. Anal., v. 22, 1985, pp. 713-728. 